TITLE: Vitamin D in Real and Simulated Weightlessness: Implications for Earth

AUTHORS: Barbara L. Rice, Sara R. Zwart, Scott M. Smith

\title{
LEARNING OUTCOME:
}

To understand the importance of dietary vitamin $\mathrm{D}$ for healthy individuals not exposed to sunlight, the difficulties of meeting vitamin D requirements with typical supplements, and the implications for other populations (such as the elderly, and patients with disease states such as cancer or diabetes).

\section{TEXT:}

Vitamin D deficiency has reemerged as a public health concern in the United States. It is also a concern for astronauts because spacecraft are shielded from ultraviolet light, leaving diet as the sole source of vitamin D. We report here the findings from four studies: one evaluation of astronauts before and after 4- to 6-month missions to the International Space Station, and the other three from a ground-based analog for space flight, long-term bed rest.

For the space flight study, blood samples were collected before the flight and within hours of landing after it. Crewmembers $(n=11)$ were provided vitamin D supplements (as cholecalciferol $(10 \mu \mathrm{g} / \mathrm{d})$ throughout the mission. The average number of vitamin D supplements reported to be consumed per week was $5.7 \pm 4.0$. The vitamin D status indicator serum 25 -hydroxycholecalciferol was $25 \%$ less after landing (48 \pm 20 ) than before flight (63 \pm 16) $(P<0.01)$.

A series of three studies was undertaken to evaluate nutritional changes during and after 60 or 90 days of $-6^{\circ}$ head-down-tilt bed rest. A total of 11 subjects ( 8 M, $3 \mathrm{~F}$; age 26-55 y) participated in the studies. Blood and urine were collected twice before bed rest and once per month during bed rest. During bed rest the average dietary intake of vitamin $D$ for the three studies was $4.84 \pm 0.16$ (study 1), $6.24 \pm 0.81$ (study 2), and $7.16 \pm 1.40$ (study 3) $\mu$ g/day. In study 1 only, subjects were given a daily supplement of $10 \mu \mathrm{g}$ vitamin D (as ergocalciferol). Data were analyzed using repeated-measures ANOVA. In the first study, 7 days after the end of the bed rest, serum 25-hydroxycholecalciferol was 30\% less than it was before bed rest $(\mathrm{p}<0.05)$. In the second and third studies, during or after bed rest the serum 25-hydroxycholecalciferol concentration was not significantly different from its concentration before bed rest.

These data demonstrate that vitamin D intake is critical for individuals not exposed to the sun. Although we studied astronauts and healthy subjects in bed rest, the implications of our results also apply to people living in northern latitudes and others who receive little exposure to sunlight, such as elderly people who seldom go outdoors. The inability of supplements to maintain vitamin D status is also an important finding, and highlights the need for careful food selection to ensure adequate vitamin D intake.

FUNDING DISCLOSURE: These studies were funded by the NASA. The Bed Rest studies were funded by the NASA Flight Analogs/Bed Rest Research Project. 